Pub. Mat. UAB

$N^{\circ} 20$ Set. 1980

Actes VII JMHL

\title{
ÀLGEBRES QUASI HILBERTIANES
}

Josep Pla Carreras, Ventura Verda Solans

Facultat de Matemàtiques

Universitat de Barcelona

\section{Abstract:}

In this paper we study the algebras obtained by having the deduction theorem on the sets of two elements, calling. them Q.H,-algebras.Adding to them the FREGE'S law, we obtain a Hilbert algebra; adding to then the law $(x \cdot y) \cdot y=(y \cdot x) \cdot x$, we obtain that thay form a variety; suposing the existence of a least element to the salee, we obtain an ortolattice wich gives a boolean algebra on an ortomodular lattice, according to the nature of implication classical on strong.

Una algebra QUASI-HILBERTIANAlQH-àlgebral és una terna $(S, \ldots, u)$, on $S \neq \emptyset i$. és una operació en $S i u \in S$, tal que, per tot $x, y, z, t \in S$ tenim

$$
\begin{array}{ll}
\text { QH.1. } & x \cdot x=u \\
\text { QH.2. } & x \cdot y=u \text { i }=u \cdot x=u \text { implica } x=y ; \\
\text { QH.3. } & u \cdot x=x ; \\
\text { QH.4. } & x(y \cdot x)=u ; \\
\text { QH.5. } & x \cdot(y \cdot z)=u \\
& x \cdot(y \cdot(z t))=u
\end{array}
$$

Immediatament deduin que $x \cdot u=u$ per tot $x \in S$.

S'estableix també facilment que

$$
\begin{aligned}
\text { i) } & x \cdot y=u \text { i } y \cdot x=u \text { implica } x \cdot y=u ; \\
\text { ii) } & x \cdot y=u \text { implica }(y \cdot z) \cdot(x \cdot z)=u i(z \cdot x) \cdot(z \cdot y)=u- \\
\text { iii) } & x \cdot(x \cdot y)=x \cdot y ; \\
\text { iv) } & x \cdot(y \cdot z)=u \text { implica } y \cdot(x \cdot z)=u ; \\
\text { v) } & x \cdot[(x \cdot y) \cdot y]=u .
\end{aligned}
$$


Donada una QH-algebra considerem el sistema clausura associat

$\ell=\{T \in S: u \in T \quad i(x, y \in T i x \in T)$ implica

$\mathrm{y} \in \mathrm{T}$;

i designem per $C$ I'operador conseqüencia associat.

Aleshores tenim:

TEOREMA 1.

Una terna $(S, \ldots, u)$ és una $Q H$-àlgebra si, $i$

només si, exsiteix un operador conseqüencia.

arbitrari en $S$ tal que, per tot $x \subseteq S i$ tot

$\alpha \subseteq S, \operatorname{card}(\alpha) \leqslant 1 \quad i$ tot $x, y \in S$, satisfà:

A 1. M.P : $x \cdot y \in C(X)$ implica $y \in C(x, x)$

A 2. T.de la deducció $y \in C(\alpha, x)$ implica

$$
x, y \in C(\alpha)
$$

A 3. $C(x)=C(y)$ implica $x=y$.

Aquest resultat generalitza la caracterització de les algebres de Hilbert - vía T. de la deducció de tipus 2 $i$ les àlgebres de tipus 1 -vía T. de ia deducuió de tipus 0. [v.verdú [1980]].

TEOREMA 2 .

En tota QH-àlgebra les condicions següents són equivalents:

$\mathrm{H} 1$.

$[x,(y, z)] \cdot[(x, y) \cdot(x, z)]=$

u ;

$\mathrm{H} 2$.

$(x,(y, z))$

$[y \cdot(x, z)]$

$=\mathrm{u} ;$

$\mathrm{H} 3$.

$(x \cdot y) \cdot[(y \cdot z) \cdot(x \cdot z)]$

$=\mathrm{u}$;

$\mathrm{H} 4$.

$(s, ., u)$ és una algebra de Hilbert.

- equivalentment, $C$ satisfà $A 1, A 3$ i

A2 amb card $(x) \leqslant 2$.

Anomenem ara QH-àlgebra de Sales una QH-àlgebra tal que $(x \cdot y) \cdot y=(y \cdot x) \cdot x$, per tot $x \cdot y \in S$

Hom constata fàcilment que $x \vee y=(x, y) \cdot y=\sup (x, y)$

$i$ per tant, tota QH-àlgebra de Sales ês supra-reticle. 
Les OH-álgebres de Sales constitueixen una VARIETAT; i.e: sôn equacionalment definibles.

Si les QH-aेlgebres de Sales tenen minim 0 tindrem una negació en $(S, \ldots, u ; 0)$

$$
x^{1}=x .0
$$

Hom constata que

$$
(s, \wedge, v: ')
$$

és un orto-reticle.

En aquest cas, si $x \cdot y=x^{\prime} \vee y$, aleshores. és la implicació feble de l'ortoreticle \{cf.Cignoli \{1977\}]. i per tant, $\left(S, \wedge, V,{ }^{\prime}\right)$ és una àlgebra de Boole.

Si $x, y=x \nrightarrow y$ aleshores $[\operatorname{cf}$. of $\operatorname{cit.}](S, A, V, 1)$ és un reicle ortomodular.

\section{Bibliografia:}

CIGNOLI, R. "Deductive Systems and Congmence Relations in Ortolattices". in Math.Logic, Proc. of the first brazilian conference. 1.977.

VERDU, $V$ "Lògiques abstractes $i$ estructures algebraigues associades". Curs de Doctorat. 1980 . 